\title{
Method for Single Intravenous Anesthetic Infusion in a Rodent Model
}

\author{
Shawn K Puri, Jing Li, Ming Xiong, Johan Reyes, Pratap R. Nadavaluru, Jiang Hong Ye, \\ Alex Bekker \\ Department of Anesthesiology, New Jersey Medical School, Rutgers, The State University of New Jersey, \\ Newark, NJ, USA \\ Email: Bekkeray@njms.rutgers.edu
}

Received 14 April 2015; accepted 23 May 2015; published 26 May 2015

Copyright (C) 2015 by authors and Scientific Research Publishing Inc.

This work is licensed under the Creative Commons Attribution International License (CC BY). http://creativecommons.org/licenses/by/4.0/

(c) (i) Open Access

\begin{abstract}
Venous catheterization, endotracheal intubation, and mechanical ventilation are necessary for performing total intravenous general anesthesia in rats. Intubation and IV cannulation of the rat is challenging because of the animals' small size and the lack of equipment specifically designed for the restricted anatomical dimensions. Here, we present methods for tail venous catheterization and intubation that are quickly learned by the provider with clinical operating room experience but lack experience in the lab. For tail venous catheterization, each rat $(n=20)$ was gently restrained in a rat chamber; its tail was placed in warm water for 10 minutes, and a 24 gauge intravenous catheter was inserted into the lateral tail vein. The catheter was fixed in place using tape and attached to a T-connector for drug administration. A bolus of propofol $(n=10)$, ketamine $(n=$ $7)$, or etomidate $(n=3)$ was administered to achieve rapid deep anesthesia. Once anesthetized, rats were intubated with the aid of a modified pediatric laryngoscope. The standard miller blade 0 was cut on each side for approximately $2 / 3$ of the total blade length to remove a total of half the width. After the ventilator was properly set, the rats' vital signs and metabolic status were monitored. Throughout the one-hour infusion, the rats' physiologic parameters were maintained within normal range. These results indicate that intravenous general anesthesia can be performed effectively and safely in small animals using the refined catheterization and intubation methods tested in this study. These techniques are easily reproducible and learned as they mimic the tools and strategies commonly used in the $\mathrm{OR}$.
\end{abstract}

\section{Keywords}

Refinement, Venous Catheterization, Endotracheal Intubation, Intravenous General Anesthesia, Rat 


\section{Introduction}

Intravenous (IV) administration of anesthetic drugs is a well-established practice in anesthetic delivery and a popular alternative to inhalational anesthesia [1]. Animal models such as the rat are extremely useful to further understand the safety and efficacy of intravenous anesthetic drugs. Venous catheterization and endotracheal intubation are necessary to conduct experiments in anesthetized rats. Catheterization allows for drug administration, while intubation allows for steady-state conditions during in vivo experiments. Performing these procedures on rats is challenging because of the animals' small size. Many methods have been described with high rates of success, many of these are not easily learned by the novice practitioner. We describe in our paper a method, by mimicking equipment commonly used in the OR, that can be quickly learned and easily replicated by a novice laboratory provider.

Rats lack readily accessible veins large enough for venipuncture. Many methods for vascular access in rats have been described in the literature, but these techniques are difficult to perform in a standard laboratory that lacks specialized equipment. Similarly, rats' small oral cavities make intubation difficult, and no standard equipment is available for rat intubation. Different rat intubation methods described in the literature include blind oral tracheal intubation and direct visualization of the trachea with or without the use of an intubation wedge [2]-[8]. The light-carrying laryngoscope has been previously shown to be helpful by allowing the direct visualization of the vocal cords [7]. Despite these techniques and devices, successfully intubating rats remains a challenge because even the smallest blade size (Miller 0) is wider than a rat's oral cavity.

When inserting a blade into the rat's oral cavity, visualization which is of the oral cavity is grossly obscured, making surveillance of the complete vocal cord difficult. As a result, investigators, especially those without experience with these techniques, may require multiple intubation attempts on the same animals. Repeated attempts on the same animal can increase the frequency of complications, such as laryngospasm, mucus plugging, airway edema, and glottic or local tissue trauma [9]. We also noted that if rats were not successfully intubated within two attempts, they would regain glottic reflexes, making subsequent intubation attempts difficult. To address the methodologic challenges associated with catheterization and intubation of rats for anesthetic studies, we describe (1) a gentle method of intravenous (IV) catheterization in conscious rats without administering any sedative medications and (2) an effective and reliable endotracheal intubation technique allowing complete visualization of the glottic opening using a modified laryngoscope blade. With our modifications, the intent is to make intubation conditions in rats similar to our daily clinical practice such as placing in "sniffing position" and having "direct visualization of the vocal cords before placement of endotracheal tube”.

\section{Methods and Materials}

\subsection{Animals and Housing}

Sprague-Dawley (SD) rats (Taconic Farm, Germantown, NY, USA), weighing 230 - $450 \mathrm{~g}$ at the start of the experiment, were housed in ventilated Plexiglas cages in a climate-controlled room $\left(20^{\circ} \mathrm{C}-22^{\circ} \mathrm{C}\right)$. Animals received food and water ad libitum. The room was illuminated on a 12-h light/dark schedule with lights on at 7:00 a.m. All experiments were performed in accordance with the guidelines of the National Institutes of Health for the Care and Use of Laboratory Animals and were approved by the Institutional Animal Care and Use Committee of Rutgers, New Jersey Medical School, Newark, New Jersey.

\subsection{Lateral Tail Vein Catherization Procedure in the Conscious Rat}

At the initiation of the experiment, each rat was gently restrained in a clear plastic rat holder (Figure 1(A)). In cases when this restraint caused stress to rats, the rat was placed in a slightly larger holder to allow more room. The slot at the back of the chamber was then opened; the rat's tail was pulled out from the lateral side of the chamber and placed in warm water (about $45-50^{\circ} \mathrm{F}$ ) for $5-10$ minutes to maximize venodilation (Figure 1(A)). A 24 gauge protective IV catheter (24G X 3/4") was then inserted into the rat's lateral tail vein.

To facilitate successful catheterization, the tail was bent slightly so the approach angle of the needle was almost parallel to the vein (Figure 1(B)). The dominant hand was used to hold the catheter while the non-dominant hand was used to grasp and position the tail. The tail was gripped between the thumb and index finger with the thumb on top and pulled back until it was slightly taut. The proximal bending created a slightly perpendicular angle at the base of the tail which prevented any rat movement from transmitting down to the tail where the IV 

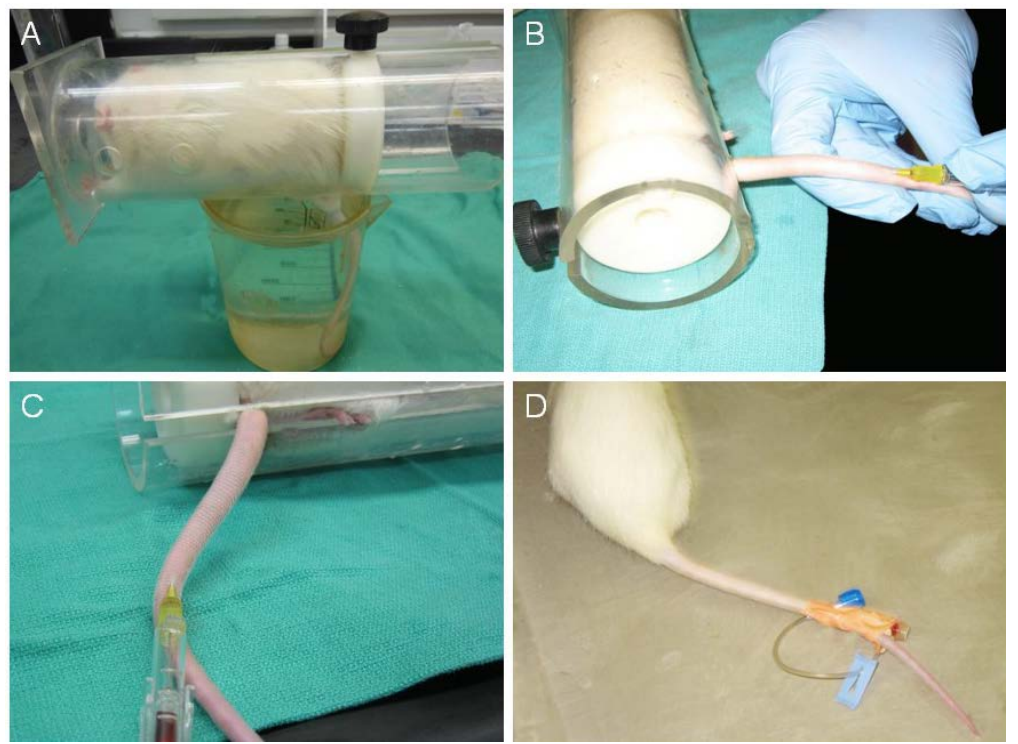

Figure 1. Procedure for inserting 24 gauge IV catheter in rat tail vein. (A) Rat tail warmed in water (45 - 50 F); (B) Animal was placed on its side and tail was bent and pulled out so IV catheter can be inserted at a flat angle into vein; (C) Blood entered the catheter upon entry of vein; (D) Needle removal: Catheter secured inplace with HY-tape and attached to T-connector extension set for drug injection or infusion.

was being inserted. The catheter needle was inserted through the skin at a flat angle and advanced for several millimeters. When the needle was successfully in the vein, blood entered the catheter (Figure 1(C)). Once blood was visualized, the needle was pulled out to avoid perforation, leaving only the plastic catheter in the vein. In cases when no blood entered the catheter but appeared to be inserted correctly, the catheter was slowly retracted along the tail while rotating the catheter until blood was seen in the catheter. Correct insertion into an unperforated vein was verified and confirmed by flushing a small amount of saline into the catheter. When the catheter was in the vein correctly, the plunger moved easily and the dark colored vein was cleared as the saline was injected. If the plunger was hard to push or the tail around the injection site blanched white, the catheter was not in the vein. Initial attempts to insert the catheter were made in the distal third portion of the tail; when needed, subsequent attempts could then be made in more proximal portions of the tail, downstream from the previous puncture sites. After confirming the catheter placement, it was secured with HY-Tape (Allegro Medical Supplies Inc., Bolingbrook, IL) and attached to a T-connector extension set (Baxter Healthcare Corporation, Deerfield, IL, USA) for drug injection or infusion (Figure 1(D)). The rats were then released to the home cage (Figure 1(D)).

\subsection{Equipment Required for Endotracheal Intubation}

Several pieces of equipment were modified to simplify the rat intubation procedure. An intubation stand was assembled using an adjustable metal book holder (Figure 2(A)). Advantageously, this setup was easily recreated when needed and served as an economical alternative to commercial rat intubation equipment. It greatly improved the rate of successful intubation by improving airway axis alignment and anterior tongue displacement. Intravenous catheters (16 or 18 gauge) removed from their original stylets were used as intubation tubes for the rats. The length of the stylet was made $1 \mathrm{~cm}$ less than the catheter length to prevent accidental trauma. A modified pediatric laryngoscope blade with attached LED light source served as the blade (Figure 2(A)). This modified laryngoscope blade was created from a standard pediatric Miller 0 blade. Half of the blade's width, extending from the blade's distal tip to approximately two-thirds of the blade's length, was removed using a metal cutting saw (Figure 2(B)). The sides were sanded until smooth and uniform. The light source and the attachment site for the handle remained intact. The laryngoscope blade was narrowed so it could be easily positioned anteriorly through the rat's neck to visualize the vocal cords.

The small animal ventilator (Harvard Apparatus, Holliston, MA) was used to ventilate the rats by maintaining 

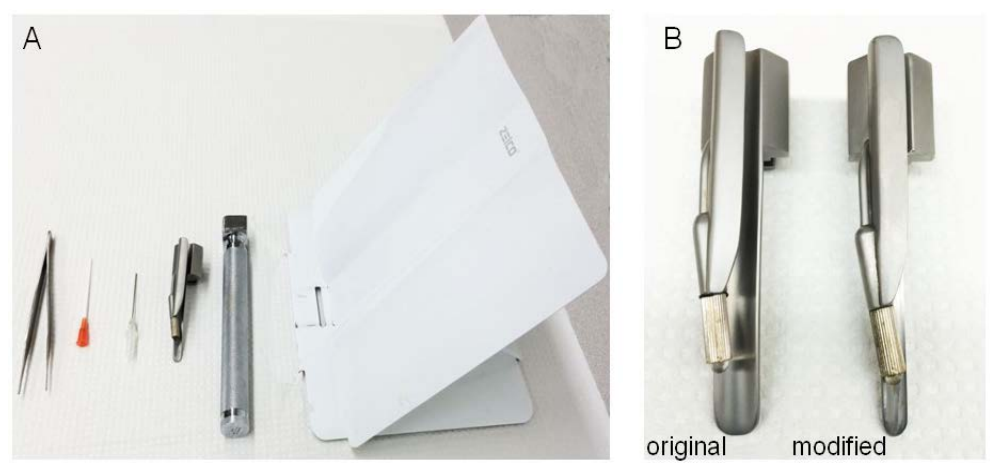

Figure 2. Intubation Tools. (A) Intubation tools: metal book holder, laryngoscope handle and modified blade, 15 gauge catheter and stylet, forceps. (B) Modified laryngoscope (right) based on standard pediatric Miller blade (left).

the guideline parameters set by the manufacturer for tidal volume, respiratory mode and rate, inspiration to expiration ratio, and inspiratory pressure. End title $\mathrm{CO}_{2}\left(\mathrm{EtCO}_{2}\right)$ was monitored by Capnomac Ultima $\mathrm{CO}_{2}$ monitor (Datex Ohmeda, Louisville, KY). Vital signs (e.g., continuous arterial oxygen saturation, pulse distension, heart rate, and breath rate) were monitored using a pulse oximeter (Harvard Apparatus, Holliston, MA). A temperature controller (Harvard Apparatus, Holliston, MA) was used to maintain the body temperature of rats at $37.5^{\circ} \mathrm{C}$.

\subsection{Animal Preperation and Intubation Process}

Prior to intubation, general anesthesia was induced with a bolus of propofol, ketamine, or etomidate. Rats lost righting reflex several seconds after receiving a bolus of anesthetic. Subject rats were then immediately positioned in a 45-degree plane and suspended by a regular ribbon hooked around the upper incisors (Figure 3(A)). The person performing the intubation procedure stood behind the inclined metal plate. Hyper salivation induced by anesthetics (especially ketamine) was occasionally noted and were cleared by a 14-gauge intravenous catheter connected to low suction.

To facilitate intubation, the animal's mouth was opened using forceps and the tongue was displaced to the side using forceps. The modified laryngoscope blade was inserted into the oral cavity (Figure 3(B)) cords were visualized by pushing the tip of the epiglottis upward with the tip of the blade. Visualizing the glottic opening, we introduced an 18 or 16 gauge IV catheter with the guide stylet through the vocal cords. Care was taken to ensure that the guide stylet did not project out of the catheter to avoid injury to the delicate organ. With the catheter placed in the trachea, the guide stylet was carefully removed and the catheter was connected to the $\mathrm{Y}$ tube of the ventilator. $\mathrm{EtCO}_{2}$ was monitored to ensure that the catheter was correctly positioned. Once its position was confirmed, the catheter was affixed to the rat's nose (Figure 3(C)). To ensure adequate oxygenation and ventilation during the apneic period following general anesthesia induction, a maximum of one minute was allotted to the provider for the intubation process.

The ventilator settings for respiration rate were 80 - 90 respirations per minute and for tidal volume 3.0 - 4.5 cc. The ventilator was connected to an oxygen tank and supplied with $1.0 \mathrm{~L} / \mathrm{min}$ of oxygen to maintain a satisfactory pulse oxygen saturation ( $>97.0 \%$ ). The respirator settings above were tested and optimized based on the rat's vital signs and metabolic studies. A temperature probe was inserted into rat's rectum. The probe was connected to a temperature controller (Figure $3(\mathrm{C})$ ) to maintain the anesthetized rat's body temperature at $37.5^{\circ} \mathrm{C}$. This intubation technique was quickly learned by members of the study team and performed in approximately two to four minutes.

\subsection{Target Control for Total Intravenous Anesthesia Procedure}

After administering a bolus of propofol, ketamine, or etomidate, the venous catheter was immediately connected to a $10 \mathrm{ml}$ syringe driven by a syringe pump (Harvard Instruments, South Natick, Mass., USA). A continuous infusion was administered to maintain an intermediate surgical plane of anesthesia (Figure 3(C)). During anesthesia, thearterial oxygen saturation, pulse distension, heart rate, and respiration rate during general anesthesia were monitored via pulse oximeter. The anesthetic plane was defined as an absence of movement and stable 


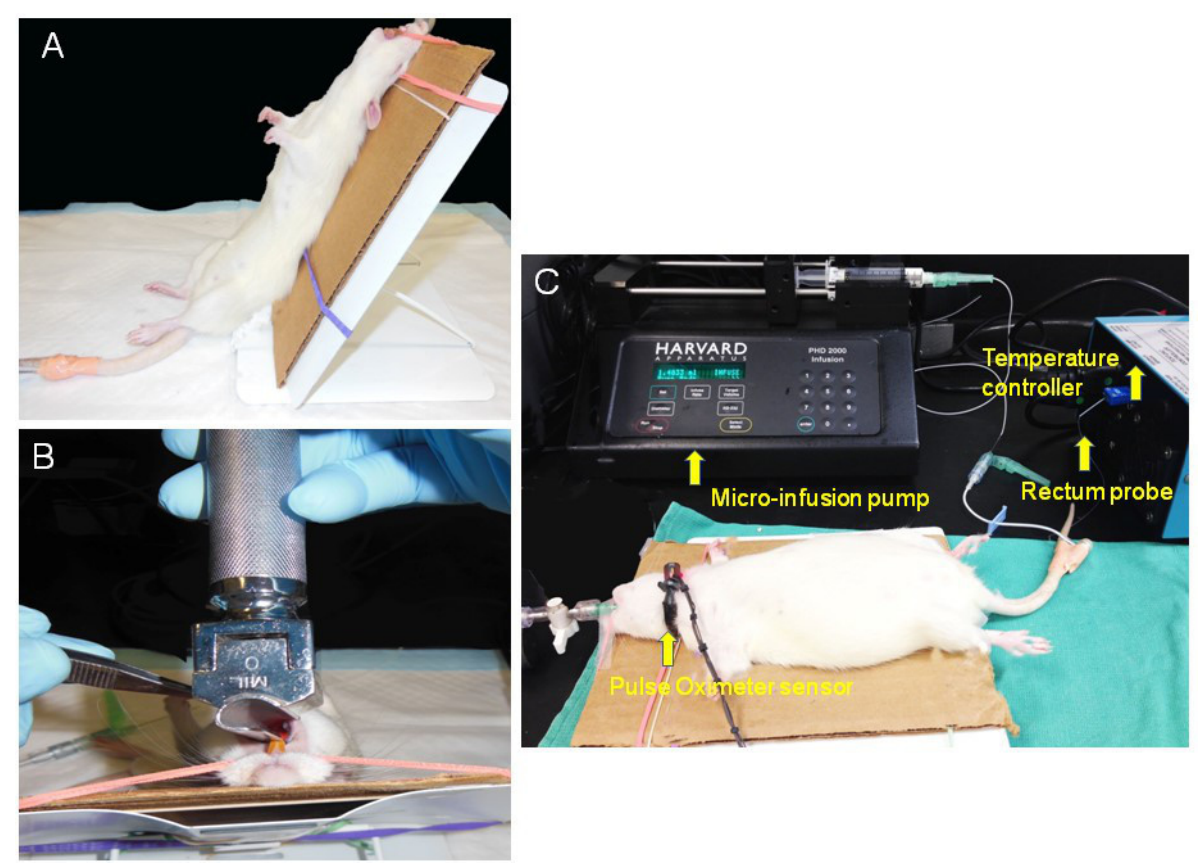

Figure 3. Endotracheal intubation and TIVA. (A) Rat positioned in semi suspended dorsal recumbency position on hard cardboard sheet which was bound to the metal book holder. The upper ribbon was hooked around upper incisors; (B) Visualization of vocal cords with modified laryngoscope; (C) Connection of endotracheal tube to ventilator and IV catheter connected to micro infusion pump for TIVA A rectal probe is in place for temperature monitoring.

changed in heart rate in response to profound mosquito-clamp pinch at hand and foot performed at 5 minute intervals (response defined as greater than $10 \%$ increase from baseline heart rate). After one hour of anesthesia, the infusion was discontinued and the rats weaned from mechanical ventilation through the assisted ventilation mode. When the rats showed signs of adequate spontaneous respiration the mechanical ventilation was stopped and the endotracheal tube was gently removed.

\section{Statistical Analysis}

Physiologic parameters were analyzed using repeated measures two-way ANOVA. Statistical significance was declared at $p<0.05$.

\section{Results}

The described procedures were tested with a total of 20 adult SD rats. After a catheter was placed in the rats' tail veins, they were allowed to move freely in their home cages (Figure 1(D)). Ten of the study rats received induction propofol $(10.0 \mathrm{mg} / \mathrm{kg}), 7$ received ketamine $(30 \mathrm{mg} / \mathrm{kg})$, and 3 received etomidate $(3.0 \mathrm{mg} / \mathrm{kg})$ to achieve rapid deep anesthesia (rapid intravenous induction) (Table 1). The duration of anesthesia induced by the anesthetic bolus lasted approximately 10 minutes, a sufficient amount of time for the experimenter to fix the rat to the intubation stand and conduct intubation. Using the modified human laryngoscope, endotracheal intubation and ventilation were successfully performed in all rats without major technical problems. To maintain an intermediate surgical plane of anesthesia, the infusion rates were $60-90 \mathrm{mg} / \mathrm{kg} / \mathrm{h}$ for propofol, $90 \mathrm{mg} / \mathrm{kg}$ for ketamine, and $10 \mathrm{mg} / \mathrm{kg} / \mathrm{h}$ for etomidate (Table 1 ).

The 20 study rats were randomized to one of three medication groups. Regardless of their group, none of the rats showed any significant change in physical parameters during anesthesia. Vital signs obtained via pulse oximeter showed that the arterial oxygen saturation was maintained at greater than $97 \%$ and heart rate and pulse distension were stable over 1 hour anesthesia with propofol, ketamine, or etomidate (Figure 4). Blood gas analysis showed stable mean $\mathrm{pH}$, oxygen and carbon dioxide. 
Table 1. Dosages of different intravenous anesthetics used to induce rapid anestheis anad maintain general anesthesia in rats.

\begin{tabular}{ccc}
\hline Agent & \multicolumn{3}{c}{ Drug Dosage } \\
\cline { 2 - 3 } & Bolus Dose $(\mathrm{mg} / \mathrm{kg})$ & Maintenance Infusion $(\mathrm{mg} / \mathrm{kg} / \mathrm{hr})$ \\
\hline Propofol $(\mathrm{n}=10)$ & 10 & $60-90$ \\
Ketamine $(\mathrm{n}=7)$ & 30 & 90 \\
Etomidate $(\mathrm{n}=3)$ & 3 & 10 \\
\hline
\end{tabular}
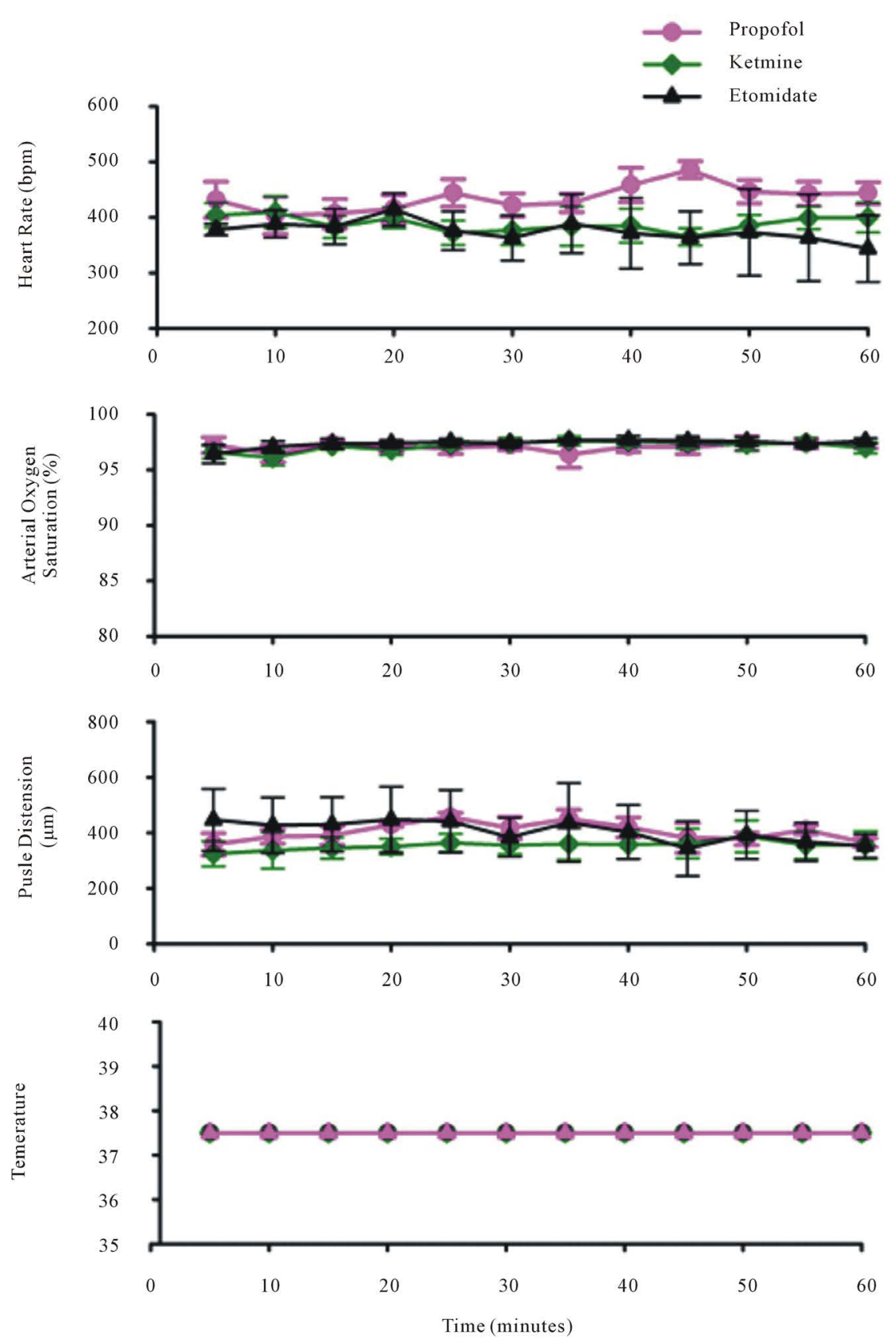

Figure 4. Vital signs of rats during TIVA. Arterial oxygen saturation, heart rate and pulse distention were monitored via pulse oximetry. The data was averaged at 5 minute intervals during TIVA. Temperature was maintained at $37.5^{\circ} \mathrm{C}$ using temperature controller. Data indicate that vital signs were stable during TIVA with propofol $(n=10)$, ketamine $(n=7)$ or etomidate $(n=3)$. Data are presented as mean \pm SEM. 


\section{Discussion}

This study demonstrates a successful, safe intravenous anesthesia rat model using refined venous catheterization and intubation techniques. Moreover, these techniques are easily learned by those with clinical anesthesia experience but with minimal knowledge of advanced laboratory techniques. In this study, one of the keys to successfully catheterizing the conscious rat's tail vein was to dilate the vein by warming the tail. In rats, the tail is a major thermoregulation organ with a large surface area available for heat loss. Therefore, warming prior to injection results in vein dilation and enhanced blood flow, which support successful venipuncture. Previous studies suggest that a rat's whole body should be warmed to approximately $40^{\circ} \mathrm{C}$ by placing the animal into a thermostatically warmed "hot-box" [10]. However, this procedure places the rats at risk for hyperthermia. The alternate procedure described in this study mitigates this risk while ensuring that good venous filling is easily achieved. Typically, researchers use butterfly needles to administer fluids via the tail vein. With this method, indwelling needles can dislodge or perforate the vein whenever the syringe is manipulated or the restrained rat flinches. Implanting a 24 gauge protective IV catheter with a percutaneous insertion method improves reliability of venous catheterization and safe medication administration.

Although commercial vascular catheters for rats and mice are available, such as restrained tail vein infusion model (Braintree Scientific Inc., Braintree, MA), our method provides similar advantages with readily available materials. The method described in the Braintree model was a nonsurgical approach where a "finder needle" punctured the vein and once free flow of blood was confirmed, a guide wire was inserted and a catheterr was then placed over this into the vein. However, it can be difficult to identify the insertion site for the guide wire after withdrawing the finder needle, especially for the inexperienced operator. In the current study, we noted that 24 gauge catheter ( $3 / 4$ " length) has two distinct advantages. First, the needle and catheter are combined, thereby eliminating the need to remove the needle once the vein is accessed. Once the needle is inserted into the tail vein through the intact skin, the catheter is simultaneously guided into the vein minimizing accidental dislodgement of catheter outside the vein. Second, it provides a visual check that the vein has been entered: blood fills the needle chamber (Figure 1(C)). Once the catheter is established intravenously (Figure 1(D)), any movement by the rat or the operator does not lead to penetration or laceration of the vessel wall as easily as a hypodermic needle. The utilization of 24 gauge IV catheter has greatly improved the likelihood of a successful implantation of the catheter in the tail vein in the conscious rats. More than $90 \%$ of successful implantation in our rats occurs in the first attempt. Moreover, it mirrors the IV placement technique used in clinical practice, making it easy to learn and minimize complications and trauma to the animal.

In the current experiment, a refined method of endotracheal intubation in rats was also described. Various authors indicate that visualizing the laryngeal opening is important in order to perform successful intubation in the rat and reduce the time of the procedure [2]-[4] [8]. A light-carrying laryngoscopic blade (laryngoscope) designed by Molthen greatly improved the success rate of intubation in rats [8]. However, creating a laryngoscope from scratch requires a variety of materials and technical skills. While a standard human laryngoscope is a good tool for endotracheal intubation in the rats [8]. We have noted that even the smallest size of infant standard laryngoscope blade is wider than the rat's oral cavity. It often obstructs the operator's view of the laryngeal cavity when attempting to insert the endotracheal tube into the trachea. In this study, we cut off half of the blade width (Figure 1(B)) to more closely approximate the anatomical dimensions of the rat's airway while the light source and the attachment site for the handle remained intact. When inserted into the laryngeal cavity, it permits securing and manipulating the tongue, while illuminating the oropharynx to yield good visualization of the arytenoid cartilage and glottis.

The intubation procedure becomes simple and quick with the help of the modified pediatric laryngoscope blade presented in this article. The time required to complete the intubation was less than 30 seconds on average. The technique was quickly learned. By scaling the size versus creating a new device, we retain the familiarity of the airway tool and the associated intubation technique. Other methods may require additional steps that the novice is unfamiliar with therefore increasing rate of initial complication when learning the technique. Moreover, many of the techniques may be considered "blind" or "partially blind" techniques that rely on operator expertise and again produce a steep learning curve.

In our model the combination of the adjustable stand which allows rapid positioning which minimizes hypoxia and apnea time and the use of the modified blade allows for a reliably unobstructed view of the glottis opening and placement of the endotracheal catheter under direct visualization. In particular, the insertion of the 
Table 2. Arterial blood gas results of rats during TIVA with Propofol.

\begin{tabular}{|c|c|c|c|c|}
\hline & \multicolumn{4}{|c|}{ During 1 hour Propofol infusion } \\
\hline & $15 \mathrm{~min}$ & $30 \mathrm{~min}$ & $45 \mathrm{~min}$ & $60 \mathrm{~min}$ \\
\hline pH (arterial) & $7.37 \pm 0.00$ & $7.38 \pm 0.02$ & $7.37 \pm 0.02$ & $7.39 \pm 0.03$ \\
\hline $\mathrm{P}_{\mathrm{A}} \mathrm{CO}_{2}(\mathrm{mmHg})$ & $44.7 \pm 2.1$ & $46.7 \pm 1.4$ & $45.6 \pm 2.9$ & $46.1 \pm 3.4$ \\
\hline $\mathrm{P}_{\mathrm{A}} \mathrm{O}_{2}(\mathrm{mmHg})$ & $282 \pm 51$ & $298 \pm 62$ & $299 \pm 51$ & $283 \pm 23$ \\
\hline $\mathrm{HCO}_{3}$ & $29.9 \pm 2.8$ & $31.3 \pm 1.5$ & $30.7 \pm 1.1$ & $28.3 \pm 0.3$ \\
\hline $\mathrm{Na}^{+}$ & $141.2 \pm 0.9$ & $140.3 \pm 0.7$ & $140.0 \pm 0.6$ & $141.3 \pm 0.9$ \\
\hline $\mathrm{K}^{+}$ & $4.7 \pm 0.2$ & $5.1 \pm 0.3$ & $5.2 \pm 0.3$ & $4.9 \pm 0.2$ \\
\hline Glucose (mM) & $136.3 \pm 10.4$ & $135.0 \pm 10.2$ & $133.7 \pm 3.3$ & $141.3 \pm 8.7$ \\
\hline $\mathrm{Hb}(\mathrm{mg} / \mathrm{dl})$ & $13.4 \pm 0.0$ & $13.3 \pm 0.3$ & $13.6 \pm 0.5$ & $13.5 \pm 0.7$ \\
\hline
\end{tabular}

Data are presented as mean \pm S.M.E.; $\mathrm{P}_{\mathrm{A}} \mathrm{CO}_{2}=$ arterial carbon dioxide tension; $\mathrm{P}_{\mathrm{A}} \mathrm{O}_{2}=$ arterial oxygen tension; Hb = hemoglobin; $\mathrm{n}=4$.

endotracheal catheter into the trachea became much easier because the modified blade allowed for refined manipulations of the upper airway while the LED light source allowed a clear, direct view of the epiglottis and vocal cords. This greatly reduced the potential for airway complications like esophageal intubation and direct trauma to oropharynx and trachea.

In the current study, we set up an intravenous anesthesia rat model using the refined venous catheterization and intubation techniques. It is well known that there are differences in anesthetic sensitivity and pharmacokinetics in different species and aging young animals. Accordingly, rodents require a higher dosage of anesthetics per body weight to achieve general anesthesia than humans and the dose of anesthetics varies slightly from animal to animal [11]. We found that propofol at the bolus dose of $10.0 \mathrm{mg} / \mathrm{kg}$, ketamine at the bolus dose of 30 $\mathrm{mg} / \mathrm{kg}$, and etomidate at the bolus dose of $3.0 \mathrm{mg} / \mathrm{kg}$, respectively, induced rapid anesthesia in adult rats (Table 1). The initial bolus dose was given to achieve an adequate depth of anesthesia for intubation with the duration of action lasting approximately 10 minutes. We also tested the infusion rate for each venous anesthetic drug to maintain a surgical plane of anesthesia in rats. Propofol at the infusion rate of $60-90 \mathrm{mg} / \mathrm{kg} / \mathrm{h}$, ketamine at the rate of $90 \mathrm{mg} / \mathrm{kg}$, and etomidate at the rate of $10 \mathrm{mg} / \mathrm{kg} / \mathrm{h}$ (Table 1) can maintain anesthesia in rats. Furthermore, the vital signs and metabolic status of dams during anesthesia were closely monitored. The results indicated that all vital signs (arterial oxygen saturation, heart rate, breathing rate) were stable during $1 \mathrm{~h}$ anesthesia with propofol, ketamine or etomidate. $\mathrm{PaCO}_{2}, \mathrm{PaO}_{2}, \mathrm{pH}, \mathrm{HCO}_{3}^{-}$and blood glucose levels remained within a physiologically acceptable range during the 1 hour propofol infusion (Table 2). The current study suggests that intravenous anesthesia can be performed safely in the rats at these dosages.

In summary, we have created an intuitive user-friendly method of tail vein catheterization and orotracheal intubation that allows for safe performance of intravenous anesthesia in the rat. The development of these methods affords the benefit of minimizing confounding variables such as hypotension, hypoxia, and acidosis. Additionally, this technique is particularly useful in specific situations that may not allow the use of more than one anesthetic agent. In our lab, these methods have served as the foundation for multiple studies investigating the neurotoxic effects on offspring exposed to propofol via the mother during the gestational period [12] [13]. Our methods can be performed in a standard laboratory and easily reproducible and learned, thereby reducing some of the inherent difficulty associated with these delicate experiments. Based on the low cost, simplicity, and reliability, these methods can be used in every laboratory.

\section{References}

[1] Sneyd, J.R. (2004) Recent Advances in Intravenous Anaesthesia. British Journal of Anaesthesia, 93, 725-736. http://dx.doi.org/10.1093/bja/aeh253

[2] Clary, E.M., O’Halloran, E.K., De La Fuentes, S.G. and Eubanks, S. (2004) Videoendoscopic Endotracheal Intubation of the Rat. Laboratory Animals, 38, 158-161. http://dx.doi.org/10.1258/002367704322968830

[3] Costa, D.L., Lehmann, J.R., Harold, W.M. and Drew, R.T. (1986) Transoral Tracheal Intubation of Rodents Using a 
Fiberoptic Laryngoscope. Laboratory Animal Science, 36, 256-261.

[4] Linden, R.D., Shields, C.B., Zhang, Y.P., Edmonds, H.L. and Hunt, M.A. (2000) A Laryngoscope Designed for Intubation of the Rat. Contemporary Topics in Laboratory Animal Science, 39, 40-42.

[5] Kastl, S., Kotschenreuther, U., Hille, B., Schmidt, J., Gepp, H. and Hohenberger, W. (2004) Simplification of Rat Intubation on Inclined Metal Plate. Advances in Physiology Education, 28, 29-32. http://dx.doi.org/10.1152/advan.00008.2003

[6] Weksler, B., Ng, B., Lenert, J. and Burt, M. (1985) A Simplified Method for Endotracheal Intubation in the Rat. Journal of Applied Physiology, 76, 1823-1825.

[7] Jou, I.M., Tsai, Y.T., Tsai, C.L., Wu, M.H., Chang, H.Y. and Wang, N.S. (2000) Simplified Rat Intubation Using a New Oropharyngeal Intubation Wedge. Journal of Applied Physiology, 89, 1766-1770.

[8] Molthen, R.C. (2006) A Simple, Inexpensive, and Efective Light- Carrying Laryngoscopic Blade for Orotracheal Intubation of Rats. Journal of the American Association for Laboratory Animal Science, 45, 88-93.

[9] Rivard, A.L., Simura, K.J., Mohammed, S., Magembe, A.J., Pearson, H.M., Hallman, M.R., Barnett, S.J., Gatlin, D.L., Gallegos, R.P. and Biano, R.W. (2006) Rat Intubation and Ventilation for Surgical Research. Journal of Investigative Surgery, 19, 267-274. http://dx.doi.org/10.1080/08941930600778297

[10] Conybeare, G., Leslie, G.B., Angles, K., Barrett, R.J., Luke, J.S. and Gask, D.R. (1988) An Improved Simple Technique for the Collection of Blood Samples from Rats and Mice. Laboratory Animals, 22, 177-182. http://dx.doi.org/10.1258/002367788780864529

[11] Larsson, J.E. and Wahlstrom, G. (1998) The Influence of Age and Administration Rate on the Brain Sensitivity to Propofol in Rats. Acta Anaesthesiologica Scandinavica, 42, 987-994. http://dx.doi.org/10.1111/j.1399-6576.1998.tb05360.x

[12] Li, J., Xiong, M., Alhashem, H.M., Zhang, Y., Tilak, V., Patel, A., Siegel, A., Ye, J.H. and Bekker, A. (2014) Effects of Prenatal Propofol Exposure on Postnatal Development in Rats. Neurotoxicology and Teratology, 43, 51-58. http://dx.doi.org/10.1016/j.ntt.2014.03.006

[13] Xiong, M., Li, J., Alhashem, H.M., Tilak, V., Patel, A., Pisklakov, S., Siegel, A., Ye, J.H. and Bekker, A. (2014) Propofol Exposure in Pregnant Rats Induces Neurotoxicity and Persistent Learning Deficit in the Offspring. Brain Sciences, 4, 356-375. http://dx.doi.org/10.3390/brainsci4020356 